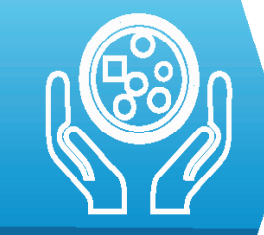

ONCOLOGY
1) Research Center for Functional Genomics, Biomedicine and Translational Medicine, Iuliu Hatieganu University of Medicine and Pharmacy, Cluj-Napoca, Romania

2) Leon Daniello Pulmonology Hospital, Cluj-Napoca, Romania

3) MEDFUTURE-Research Center for Advanced Medicine, Iuliu Hatieganu University of Medicine and Pharmacy, Cluj-Napoca, Romania

4) Department of Functional Genomics and Experimental Pathology, "Prof. Dr. Ion Chiricuta" Oncology Institute, Cluj-Napoca, Romania

5) Department of Surgery, Iuliu Hațieganu University of Medicine and Pharmacy, Cluj Napoca, Romania

6) University of Oradea, Oradea, Romania

DOI: $10.15386 / \mathrm{mpr}-1725$

Manuscript received: 14.05 .2020

Received in revised form: 14.07.2020

Accepted: 31.07.2020

Address for correspondence:

ioan.simon@umfcluj.ro

This work is licensed under a Creative Commons Attribution-NonCommercialNoDerivatives 4.0 International License

\section{EBUS in optimizing non-small cell lung cancer diagnosis and treatment}

Antonia Haranguș ${ }^{1,2}$, Ioana Berindan-Neagoe ${ }^{1,3,4}$, Lăcrămioara Toma ${ }^{2}$, Ioan Șimon ${ }^{5}$, Ovidiu Pop ${ }^{6}$, Mărioara Șimon ${ }^{2}$

\begin{abstract}
Background. Endobronchial ultrasound-guided transbronchial needle aspiration (EBUS-TBNA) is a commonly used minimally invasive method for the diagnosis and staging of lung cancer. In order to improve its diagnostic accuracy, rapid onsite cytologic evaluation (ROSE) is being utilized in some institutions. ROSE, performed by a cytopathologist in the examination room, allows the assessment of the adequacy of the collected samples, identifies malignant cells and sometimes establishes diagnosis on the spot, thus improving diagnostic sensitivity. As nonsmall cell lung carcinomas (NSCLC) require not only pathological subtyping, but also molecular characterization, obtaining the adequate amount of tissue is crucial. Only a limited number of studies have analyzed the suitability of EBUS-TBNA samples for assessment of epidermal growth factor receptor (EGFR), anaplastic lymphoma kinase (ALK) and programmed death-ligand 1 (PD-L1) status.

Aim. We intended to examine the diagnostic yield of ROSE in NSCLC and the results and feasibility of molecular analysis performed on EBUS-TBNA small samples.

Methods. 100 patients with lung tumors and hilar and/or mediastinal lymphadenopathy on $\mathrm{CT}$ or $\mathrm{PET/CT}$ scans were retrospectively identified over a 3-year period, from a prospectively maintained EBUS-TBNA database. All examinations were accompanied by on-site cytological exam - ROSE, histopathological exam (HPE) and, in the case of NSCLC, molecular testing. After the sampling of the lymph nodes, specimens were Diff-Quik stained and a rapid preliminary diagnosis was established. Immunohistochemistry and mutational testing were performed using cell blocks.
\end{abstract}

Results. Adenocarcinoma was the most frequent diagnosis in both ROSE (34\%) and histopathology (53\%). Overall sensitivity and positive predictive value of ROSE in NSCLC, considering HPE the gold standard, were $92.18 \%$ and $93.65 \%$, respectively, with a specificity and negative predictive value of $75 \%$ and $70.58 \%$, respectively. All samples that were tested for EGFR mutation and ALK rearrangement were adequate for analysis. The adequacy ratio for PD-L1 was $91.66 \% ; 37.5 \%$ of patients showed a high PD-L1 expression level, with a tumor proportion score TPS $\geq 50 \%$.

Conclusion. EBUS-TBNA is a valuable method for lung cancer diagnosis. ROSE proved to have a moderate prediction of the final diagnosis in NSCLC. Molecular analysis of EGFR, ALK and PD-L1 can be successfully accomplished on EBUSTBNA small tissue samples.

Keyword: EBUS-TBNA, ROSE, NSCLC, EGFR, ALK, PD-L1 


\section{Background and Aims}

Even if the epidemiology varies worldwide, lung cancer continues to represent one of the main causes of cancer-related deaths, both in men and women. The incidence and mortality closely follow the pattern of tobacco use [1]. The Globocan statistics in 2018 ranked lung cancer as number one cancer site in incidence, mortality and prevalence in Romania, with a percentage of $20.2 \%$ cancer-related deaths [2].

With the growing incidence of the adenocarcinoma histology and the recent advances in molecular profiling and targeted therapy comes the necessity of molecular phenotyping the non-small cell lung carcinomas (NSCLC). Testing for EGFR, EML4 (Echinoderm microtubuleassociated protein like-4) and ALK fusion, ROS1 (ROS proto-oncogene 1) and PD-L1 in patients with advanced NSCLC is mandatory in most of the European countries. The first druggable target identified in NSCLC was the EGFR mutation, with a higher prevalence reported in Asian population, females and never-smokers. Deletions in exon 19 and substitutions in exon 21 can be observed in almost $90 \%$ of cases and have been correlated with improved progression-free survival to first- and second-generation EGFR tyrosine kinase inhibitors (TKIs) [3,4]. Between $2-7 \%$ of the NSCLC patients develop ALK rearrangements, with EML4-ALK fusion oncogene being the most encountered one. This chromosomal rearrangement, which determines an aberrant downstream signaling, is successfully targeted by crizotinib, a potent TKI of ALK, that can also target hepatocyte growth factor receptor(MET) and ROS1 [5]. Second and third generation ALK inhibitors, such as ceritinib, alectinib, lorlatinib and brigatinib have rapidly followed crizotinib, also showing promising antitumor activity [6]. Moreover, the approval of three immunotherapies since 2015 in the first- and second-line treatment of patients with advanced NSCLC has rendered PD-L1 testing compulsory. A link between the degree of PD-L1 expression and the response to immunotherapy has been reported. Patients with a PD-L1 tumor proportion score $\geq 50 \%$ will have a better response to pembrolizumab [7]. All these data point to the high demand for minimally invasive diagnostic methods that can provide enough tissue for ancillary studies, including immunohistochemistry and molecular testing.

Endobronchial ultrasound-guided transbronchial needle aspiration (EBUS-TBNA) is a minimally invasive method that combines direct visualization of the airways with ultrasound-guided transbronchial needle aspiration of mediastinal or hilar lymphadenopathy, or of extraluminal tumors located in direct contact with the airways. The advantage of this method is that it can provide both diagnosis and staging of lung cancer at the same time. Often, it is the sole procedure that can be performed in order to obtain tissue for diagnosis. A meta-analysis on 9 studies that included 1066 patients reported a pooled sensitivity and specificity of $90 \%$ and $99 \%$ respectively of EBUSTBNA in the diagnosis of lung cancer [8]. EBUS can be accompanied by rapid on-site cytology that could increase diagnostic accuracy, ensure sufficient material for ancillary studies, reduce the total time of the procedure and cost [9]. Data concerning the impact of ROSE on diagnostic yield is still contradictory. Regarding mutational characterization of NSCLC, EBUS-TBNA samples could be feasible for testing, but information regarding the exact number of passes needed to obtain adequate tissue is not well defined [10]. Adequacy of EBUS-TBNA for biomarker testing is reported to be between $70-95 \%$ [11]. A recent study found an adequacy ratio of $88.6 \%$ and $93.8 \%$ for EGFR mutation, respectively ALK rearrangement [12]. Regarding PD-L1 testing on small samples obtained via EBUS, this method can be a promising one as it preserves the morphological features intact and obtains an adequate number of tumor cells for analysis [13].

The aim of the present study is to present the experience of our institution in using EBUS-TBNA for the diagnosis of NSCLC, to evaluate the diagnostic yield of ROSE in NSCLC and the feasibility of molecular testing performed on EBUS-TBNA tissue samples.

\section{Methods}

This is a retrospective study on patients admitted to the Pulmonology Hospital of Cluj-Napoca, Romania, between January 2017 and March 2020, with a suspicion of lung cancer on computed tomography (CT scan) or positron emission tomography/CT (PET/CT), in order to be diagnosed by EBUS-TBNA in the Bronchology Department. Out of a total of 311 patients who presented with different pathologies and required EBUS-TBNA during this period, we identified, from our prospectively maintained database, 100 patients with lung tumors and hilar and/or mediastinal lymphadenopathy. All patients were initially evaluated using flexible bronchoscopy under local anesthesia, but this procedure could not obtain tissue for the diagnosis of lung cancer. All EBUS procedures were performed under general anesthesia using propofol and remifentanil, with airways secured by a rigid bronchoscope or a laryngeal mark airway, depending on the patients' anatomical characteristics. A flexible Fuji EBUS-scope with an integrated ultrasound convex probe transducer (EB-530US) and 21-gauge needles were used to sample suspect lymphadenopathy with a size larger than $5 \mathrm{~mm}$. The number of needle passes per lymph nodes station and number of stations sampled were decided by the experienced operator and were recorded along with patient demographics, on-site cytological (ROSE) and final histopathological diagnoses.

Rapid on-site evaluation (ROSE) was available for every procedure. The smears were air-dried and Diff-Quik stained; 10 to 12 cytological specimens were examined. Feedback confirmed adequacy of sample and presence or 
absence of tumor cells. The remaining specimens were fixed in $95 \%$ alcohol, sent to laboratory and stained with Papanicolaou staining and Periodic Acid- Schiff (PAS) when adenocarcinoma or squamous cell carcinoma was suspected. A lymphocytes ratio of more than $30 \%$ of nucleated elements was considered representative at ROSE. The "core" fragments were first flushed into saline filled containers, then fixed in buffered neutral formalin and embedded into paraffin. Cell blocks were examined by experienced pathologists at an offsite laboratory. Immunohistochemical staining was used in all cases. Diagnosis of adenocarcinoma was based on cytomorphology and expression of cytokeratin 7 (CK7), thyroid transcription factor (TTF)-1, with negative CK5 and p63. For the diagnosis of squamous cell carcinoma, morphology and CK5 and p63 expression were used. NSCLC-NOS was diagnosed in case of negative findings for TTF-1, CK5/6 and p63 on IHC. If the preliminary result was consistent with adenocarcinoma, the pathologist decided if the sample for adequate for subsequent EGFR, ALK and PD-L1 testing, and if it was consistent with squamous cell carcinoma, for PD-L1 determination only.

For activating EGFR mutations analysis (exons 18-21) on small biopsies, 5-10 $\mu \mathrm{m}$ sections were prepared from the paraffin block, of which two of them were hematoxylin and eosin (H\&E) stained, in order to evaluate the existence and quantity of tumor cells. Deparaffination of the slides was done using xylene and $100 \%$ alcohol. Tumor regions marked on the H\&E slide were scraped into microtubes and proteinase $\mathrm{K}$ was used to digest the tissue. The following steps included DNA extraction, use of wild-type blocking PCR (WTB-PCR), then sequencing of WTB-PCR products and the final analysis of the sequencing results. If EGFR mutations in exons 18-21 were negative, cell blocks were evaluated for ALK mutation using immunohistochemistry (D5F3).
More than 50 viable tumor cells are required for the use of IHC. A high PD-L1 expression is expressed as a Tumor Proportion Score (TPS) $\geq 50 \%$ on PD-L1 immunocytochemical stained slides (22C3 DAKO clone). More than 100 viable cells are required for assessment.

Descriptive statistics were performed using Microsoft Excel. Contingency tables were used to determine the diagnostic yield of ROSE, in comparison to the histopathological examination, the current "gold standard".

\section{Results}

Out of 311 patients that had undergone EBUSTBNA for various pathologies, 100 had parenchymal lung tumor with associated mediastinal and/or hilar lymphadenopathy on imaging studies (CT or PET/CT). Table I presents the patient demographics, characteristics of lesions on imaging and site of the targeted lymphadenopathy on EBUS-TBNA. The male to female ratio was 2.22, with 69 men and 31 women included in the study. The mean age of the group was 62.96 . The majority of patients $(71 \%)$ came from urban areas. Regarding smoking history, $68 \%$ were current smokers, while only $27 \%$ were never smokers.

Around one third of patients (36\%) had lymphadenopathy of less than $20 \mathrm{~mm}$ in short diameter on imaging. Only 7 patients had performed a PET-CT prior to presentation for the evaluation of lymph node metastasis. Most of lymph nodes targeted by EBUS-TBNA were situated in station 7 (subcarinal node), station $4 \mathrm{R}$ (right paratracheal node) and 10R (right hilar node). Figure 1 presents the ultrasonographic image of stations $4 \mathrm{R}$ and 7. The median number of needle-passes per lymph node was 4. More than two-thirds of the parenchymal lung tumors were situated on the right lung.
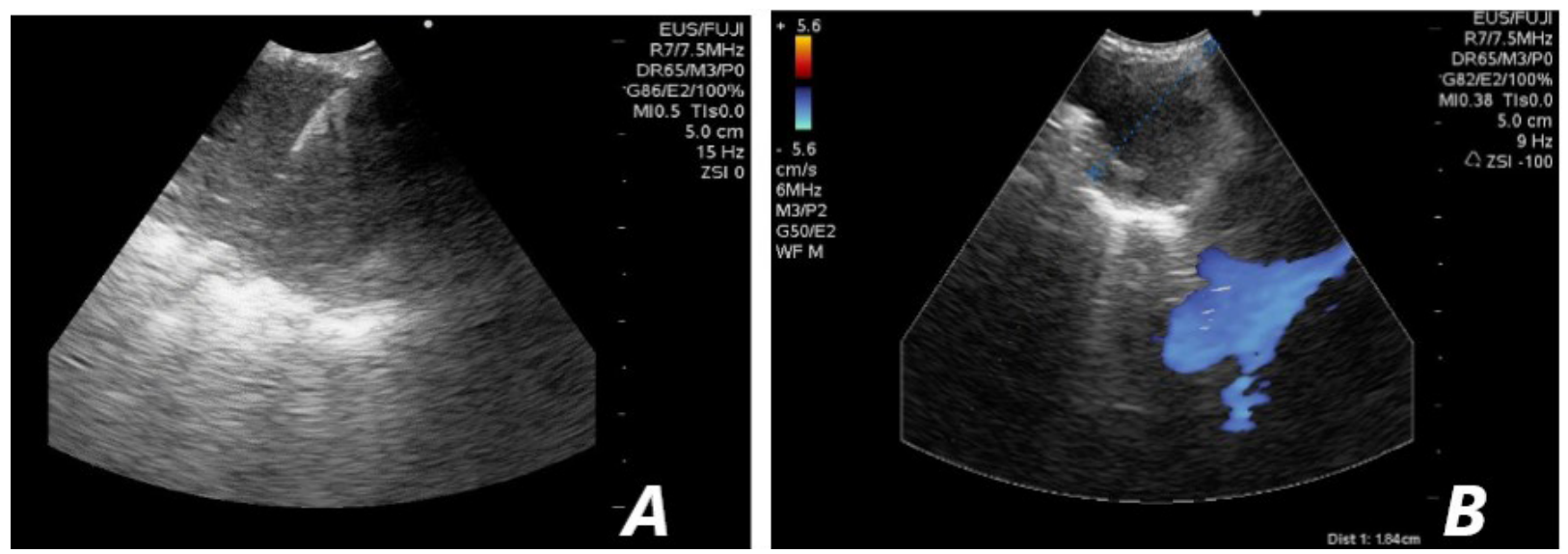

Figure 1. Ultrasonographic aspects of EBUS showing subcarinal node with hyperechoic aspiration needle (A) and inferior right paratracheal node with superior vena cava in color Doppler (B). 
Table I. Patient demographics and characteristics of the lesions.

$\begin{array}{lc}\text { Patients } & 100 \\ \text { Male/Female } & 69 / 31 \\ \text { Mean age (years) + SD } & 62.96 \pm 10.26 \\ \text { Urban/Rural } & 71 / 29 \\ \text { Smoking history } & 27 / 68 / 5 \\ \quad \text { Never-/current-/ex-smoker } & \\ \text { Lesion size } & 36 / 64 \\ \quad \text { Mean, }<20 / 220 \mathrm{~mm} & 7 \\ \text { Metabolic activity on PET-CT } & \\ \text { Location of targeted lymph nodes } & \\ 4 R & 42 \\ 4 L & 18 \\ 7 & 51 \\ 10 R & 28 \\ 10 L & 10 \\ 11 R & 8 \\ 11 L & 5 \\ \text { Side of primary suspected lesion } & 73 / 27 \\ \text { Right/left } & \end{array}$

All 100 procedures were accompanied by rapid on-site evaluation (ROSE) in the EBUS operatory room. Table II comprises the distribution of ROSE results for these patients. A precise diagnosis of malignancy could be achieved with ROSE in $80 \%$ of cases. The most frequently identified pathology was adenocarcinoma, with $34 \%$ of the patients presenting large vacuolated cells in clusters or acinar arrangements, with a high nucleus to cytoplasm ratio on ROSE (Figure 2). 2 patients had a cytological diagnosis of squamous cell carcinoma with an image of large cohesive cell with dense cytoplasm, palisading of the nuclei and presence of spindle cells. In 34\% of the cases, the cytopathologist could provide a quick diagnosis of malignancy, by identifying tumoral cells, but in $18 \%$ of the patients, no malignant cells could be seen.

Table II. Distribution of ROSE results.

\begin{tabular}{l|c} 
ROSE results & Percentage (\%) \\
Malignant cells & $34 \%$ \\
Non-small cell lung cancer & $3 \%$ \\
Adenocarcinoma & $34 \%$ \\
Squamous cell carcinoma & $2 \%$ \\
Small cell carcinoma & $6 \%$ \\
Lymphoma & $3 \%$ \\
Benign & $18 \%$ \\
Total & $100 \%$
\end{tabular}

The final histopathology results, after immunohistochemical staining, are presented in table III. NSCLC was identified in 63 patients, the most common subtype being adenocarcinoma, in $53 \%$ of the total number of patients, followed by squamous cell carcinoma in $6 \%$. In $14 \%$ of cases, a final diagnosis of small cell carcinoma could be established. In one fifth of the patients a final diagnosis of malignancy could not be established.

Table III. Distribution of histopathology results.

\begin{tabular}{lc} 
Histopathology results & Percentage (\%) \\
\hline Adenocarcinoma & $53 \%$ \\
Squamous cell carcinoma & $6 \%$ \\
Small cell carcinoma & $14 \%$ \\
Carcinoma not otherwise specified (NOS) & $4 \%$ \\
Lymphoma & $2 \%$ \\
Thymoma & $1 \%$ \\
Benign & $20 \%$ \\
Total & $100 \%$
\end{tabular}
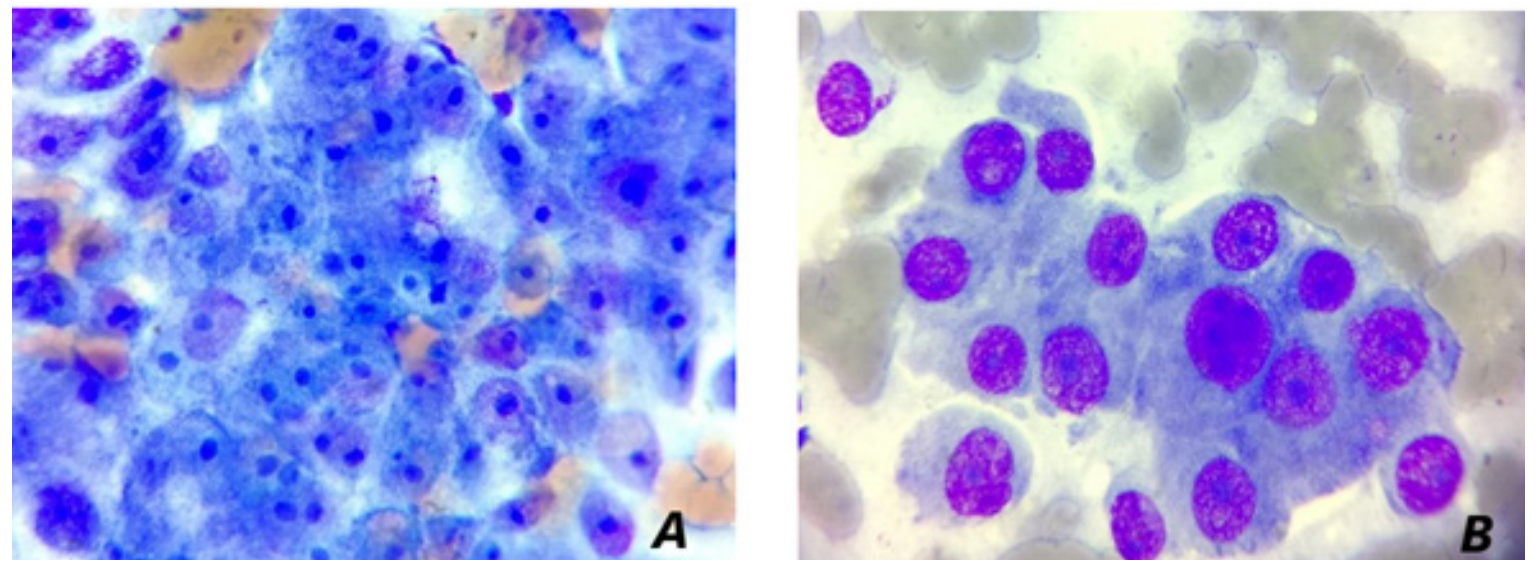

Figure 2. Adenocarcinoma aspect on ROSE (A and B): tumor cells in placards with a morular aspect, eccentric nucleus, anisokaryosis and vacuolated cytoplasm (100x Diff Quick). 

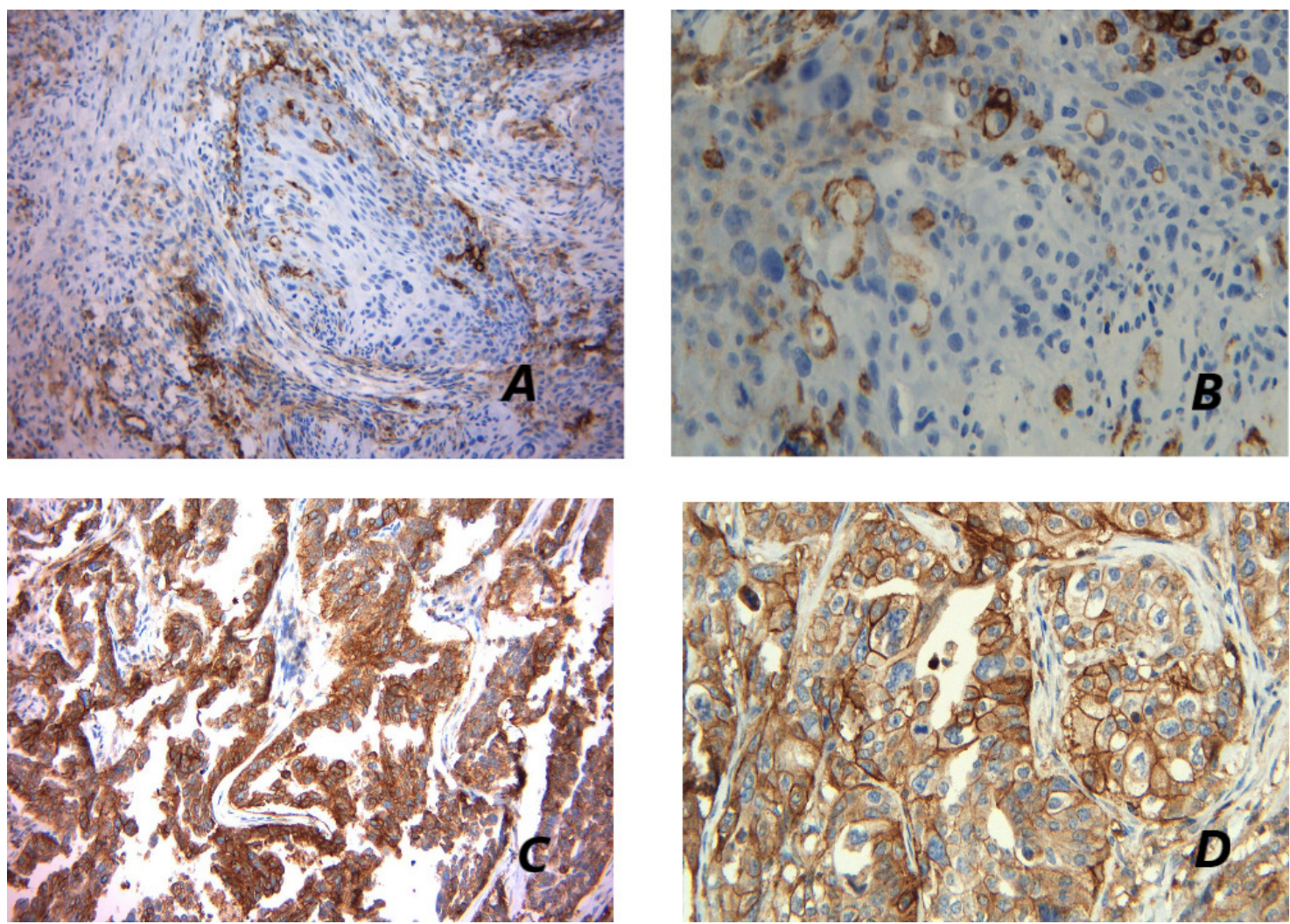

Figure 3. Examples of PD-L1 immunocytochemical staining on EBUS-TBNA cell blocks using 22C3 DAKO clone. A and B - TPS 1\% to $49 \%$ (200x magnification/400x magnification), $C$ and D - TPS $\geq 50 \%$ ((200x magnification/400x magnification).

The diagnostic yield of ROSE in NSCLC was calculated by using contingency tables and the histopathological exam as the "gold standard" diagnostic method. Table IV contains the calculated values. ROSE for the studied samples of NSCLC had a high sensitivity of $92.18 \%$, a specificity of $75 \%$, a positive predictive value of $93.65 \%$ and negative predictive value of $70.58 \%$.

Table IV. Diagnostic yield of ROSE in NSCLC.

$\begin{array}{lc}\text { Sensitivity (\%) } & 92.18 \% \\ \text { Specificity (\%) } & \mathbf{7 5 \%} \\ \text { Positive predictive value (\%) } & \mathbf{9 3 . 6 5 \%} \\ \text { Negative predictive value (\%) } & \mathbf{7 0 . 5 8 \%}\end{array}$

Table $\mathrm{V}$ comprises a summary of the mutation analysis on EBUS small specimens. Successful EGFR testing was performed in 22 patients with adenocarcinoma, since molecular testing on EBUS specimens only started in August 2018 in our institution. Two of the analyzed specimens harbored EGFR mutations (9.09\%), namely L858R substitution in exon 21 and E746-A750del in exon 19. No ALK mutations were detected on the examined EBUS specimens using IHC, but all samples were eligible for mutation analysis. Tissue specimens from 24 patients were initially considered eligible for PD-L1 mutation analysis, but 2 tests were canceled due to insufficient malignant cells (specimen cellularity is presented in Figure 3); 22 samples contained $>100$ tumor cells. Among these patients with an adequate sample, 9/24 (37.5\%) presented a high PD-L1 expression, with TPS $\geq 50 \%$, and $8.33 \%$ had a low PD-L1 expression. The adequacy ratio for PD-L1 was $91.66 \%$.

Table V. Mutation analysis of EBUS specimens.

$\begin{array}{ll}\text { Mutation } & \text { Number of patients } \\ \text { EGFR } & \mathbf{2 2} \\ \text { Positive } & 2: \text { L858R, E746-A750del } \\ \text { Wild type } & 20 \\ \text { ALK } & \mathbf{2 0} \\ \text { Positive } & 0 \\ \text { Negative } & 20 \\ \text { PD-L1 } & \mathbf{2 4} \\ \text { Positive } & 12 \\ \quad \text { TPS } \geq 50 \% & 9 \\ \text { TPS }<50 \% & 2 \\ \text { Negative } & 10 \\ \text { Not enough tissue for } & 2 \\ \text { analysis } & \end{array}$




\section{Discussion}

The approval of immunotherapy and target therapies, namely EGFR and ALK tyrosine kinase inhibitors (TKIs), for the treatment of advanced NSCLC, has increased the need for obtaining enough tumoral tissue and for conserving it with each step of the pathological diagnosis, in order to have sufficient material for all the ancillary studies. We attempted to demonstrate the feasibility of mutation analysis on small tissue specimens obtained by the use of EBUS-TBNA.

ROSE represents an important tool, since it can not only provide information about the quality of the tissue obtained, but also shorten the time of the EBUS-TBNA procedure, by reducing the number of punctures per lymph node. It also lowers the necessity for additional procedures in order to reach a diagnosis [14]. In our study, ROSE had a high sensitivity and positive predictive value for NSCLC diagnosis $(92.18 \%$, respectively $93.65 \%)$, and a moderate specificity and negative predictive value (75\%, respectively $70.58 \%$ ), making it a feasible method for screening purposes. These results are consistent with other studies regarding the diagnostic yield of ROSE, studies that report a sensitivity between $86 \%$ and $100 \%$ for the diagnosis of lung cancer [14-17]. These values demonstrate the ability of ROSE to moderately predict the final result in cases of lung cancer suspicion.

There is only one prospective randomized clinical trial concerning the use of ROSE conducted by Oki et al [14]: 120 patients with mediastinal lymphadenopathy and suspicion of lung tumor that required EBUS-TBNA for diagnosis were randomized into two groups, with or without ROSE. The samples were evaluated by a cytopathologist after the material was air-dried and stained using the DiffQuik method. This trial demonstrated a reduction in number of punctures and the necessity for additional diagnostic and staging methods with the help of ROSE. However, two larger, but retrospective studies, on 780 patients [18], respectively 294 cytology specimens [19], reported no significant difference in diagnostic yield of EBUS-TBNA with ROSE in lung cancer patients. A recent study by Mehta et al. [20] concluded that in patients with a negative result at ROSE, the 21/22G EBUS needle might not be sufficient, and an EBUS-guided forceps biopsy of the mediastinal or hilar lymph nodes could increase the diagnostic yield of the procedure. However, Simon et al. reported that ROSE could confirm the existence of tumoral cells in $81 \%$ of patients, thus shortening the total time of the procedure [21].

Regarding the final histopathological result, adenocarcinoma had the highest prevalence in our study, i.e. $53 \%$, followed by small cell carcinoma with $14 \%$, squamous cell carcinoma with $6 \%$ and NOS with $4 \%$. The high rate of lung adenocarcinoma diagnosed by EBUS-TBNA could be related to the fact that this pathology has a more peripheral localization in the lung parenchyma, therefore it is harder to diagnose by conventional bronchoscopy. Also, in comparison to squamous cell carcinoma, which mostly affects central airways and has earlier symptoms, adenocarcinoma has a lower clinical impact [22]. $20 \%$ of patients had a negative result on the final pathology result. Out of these patients, those with a persistent high suspicion of malignancy were referred to CT-guided biopsy or mediastinoscopy. An issue surrounding EBUS-TBNA is that non-diagnostic results range from 7 to $27 \%$ in literature [23] with unsatisfactory specimens usually containing no or inadequate tissue material for evaluation.

Our study showed that the small samples obtained by EBUS-TBNA can be successfully used for the molecular characterization of NSCLC by means of cell blocks. All samples tested for EGFR mutations and ALK translocation were considered adequate for testing. Testing has not been performed on all patients with adenocarcinoma in our study, because the testing started later than the chosen date to include patients subjected to EBUS. This is due to the fact that more time was necessary to gain proper experience to perform accurate testing on the small EBUS specimens. Two patients $(9.09 \%)$ with adenocarcinoma harbored an EGFR mutation, namely L858R substitution and E746-A750del. The frequency is slightly lower than those reported in previous studies of EGFR testing on small specimens that were between $10 \%$ and $47.9 \%$ [2426]. This is probably because of the smaller size of this study, owing to a more recent start of molecular testing on EBUS samples. However, there was a high success rate for EGFR and ALK testing, that might be due to the usage of cell blocks, as recommended by the College of American Pathologists [27]. The advantage of testing on cell blocks over smear preparations comes from a better correlation with tumoral cell content and a possible better conservation of tissue for ancillary studies. On the other hand, although ALK testing on cell block material is the current "golden standard", a few organizations such as the Association for Molecular Pathology or the International Association for the Study of Lung Cancer have recently acknowledged the use of ALK FISH (fluorescence in situ hybridization) testing on cytologic smears [28]. Evidence indicates that ALK FISH testing on cytological slides could avoid nuclear truncation and better preserve DNA, providing a high sensitivity to this method. Until now, our protocol has not included mutation testing on cytological samples, but has used cell block immunohistochemistry for ALK testing.

As EGFR mutation and EML4-ALK rearrangement represent mutually exclusive alterations, FISH testing for ALK could be performed for lung adenocarcinomas with wild-type EGFR [29]. That is also the current protocol for testing in our institution. As only EGFR-TKI and ALKTKI treatments are approved in our region, KRAS (Kirsten Rat Sarcoma) and ROS1 mutation testing is not currently recommended in our daily practice.

The evaluation of PD-L1 expression on the EBUSTBNA samples collected from our patients was successful in $91.66 \%$ of cases in which it was attempted. This high 
adequacy ratio is in concordance with other previous studies on small EBUS samples [30-31]. Among the 22 patients in whom testing was feasible, $40.9 \%(9 / 22)$ had a high PD-L1 expression, with a TPS $\geq 50 \%$. This percentage was higher than in most previous studies [30-31], with the exception of a recent study by Smith et al. [32] that reported $48.2 \%$ of patients with a TPS $\geq 50 \%$ on PDL1 testing on EBUS-TBNA samples. $8.33 \%$ of NSCLC samples were insufficient for molecular testing in our study. The variability of the PD-L1 levels among different studies could be related to temporal and spatial heterogeneity of PDL-1 expression, that has been previously described [33]. Regarding the influence of needle size on molecular testing, the Chest guidelines recommend the use either 21 or $22 \mathrm{G}$ needles, with a median of $\geq 3$ needle passes [10]. All needle aspirations in the present study were performed with the use of the $21 \mathrm{G}$ needle, with a median of 4 passes per lymph node. The median number of 4 passes is a grade $2 \mathrm{C}$ recommendation [34].

With regards to the benefits of ROSE in securing adequate tissue for ancillary studies following EBUSTBNA, Trisolini et al. concluded in a randomized controlled study that the use of ROSE improved the success rate of molecular testing by $10 \%$ and decreased the need for rebiopsy [35]. Doxtader et al. also proved the advantages of ROSE for PD-L1 testing on EBUS-TBNA specimens on 53 patients [36].

The possible limitations of our study are the small number of patients that have been tested for molecular alterations, the fact that no testing was provided for patients with NOS, since testing has started later that patients with NOS had been diagnosed with the help of EBUS, and no ALK mutations were detected so far using immunohistochemistry. We cannot disregard a potential consequence of tumor heterogeneity that might have led to unrepresentative samples for the lung tumor as a whole. Furthermore, the small number of patients that presented with a pathological image on PET-CT could be connected to the fact that in our country, only patients with suspected mediastinal metastases from extrapulmonary cancers of patients with solitary pulmonary nodules benefit from free of charge PET-CT investigation.

The future for the therapy of NSCLC, among other tumors, lies in its genomic landscape. microRNAs, as an example, are 19-25 nucleotide long ncRNAs involved in all hallmarks of cancer [37] and the alteration of their expression levels is correlated with development and spread of lung cancer [38]. Isolation of DNA, RNA and analysis of miRNA expression can be performed on EBUS-TBNA small samples and could be important in future genomic research in lung cancer $[39,40]$. For a great number of patients, EBUS is the least invasive method for obtaining tumoral cells and therefore could have great potential in the discovery of novel mechanisms related to lung cancer diagnostic and therapy.

\section{Conclusions}

EBUS-TBNA has proved its great value as a minimally invasive technique used for the diagnosis and staging of NSCLC. ROSE can increase this value by rapidly obtaining a preliminary diagnosis, by reducing the time of the examination, the number of punctures per lymph node and by providing an aid in securing adequate tissue sampling for molecular testing. Our results regarding ROSE show that this method has a moderate prediction of the diagnosis when suspecting NSCLC.

This is the first report regarding the efficacy of mutational analysis on EBUS-TBNA small samples, obtained from patients with NSCLC, in Romania. Molecular marker testing in NSCLC is now standard of care and is indispensable in order to tailor the best management to the patient's tumoral characteristics. The strategy of testing is determined by local availability and is mostly performed in highly specialized centers. In our study, the great proportion of adenocarcinoma has allowed us to prove that EBUSTBNA with ROSE can not only provide sufficient tissue for an accurate subtyping of the lung tumors with the use of immunohistochemistry markers, but also ensure that the remnant specimens can be successfully tested for EGFR, ALK and PD-L1. This success highly depends on a good cooperation between the members of the multidisciplinary team formed by the bronchoscopist, the anesthetist, the cytopathologist, the pathologist and the medical nurses.

\section{References}

1. Barta JA, Powell CA, Wisnivesky JP. Global Epidemiology of Lung Cancer. Ann Glob Health. 2019;85:8.

2. Fact Sheets by Cancer. Globocan.iarc.fr. 2019. Available from: https:/gco.iarc.fr/today/data/factsheets/ populations/642-romania-fact-sheets.pdf

3. Lynch TJ, Bell DW, Sordella R, Gurubhagavatula S, Okimoto RA, Brannigan BW, Harris PL, et al. Activating mutations in the epidermal growth factor receptor underlying responsiveness of non-small-cell lung cancer to gefitinib. $\mathrm{N}$ Engl J Med. 2004;350:2129-2139.

4. Rosell R, Carcereny E, Gervais R, Vergnenegre A, Massuti B, Felip E, et al. Erlotinib versus standard chemotherapy as first-line treatment for European patients with advanced EGFR mutation-positive non-small-cell lung cancer (EURTAC): a multicentre, open-label, randomised phase 3 trial. Lancet Oncol. 2012;13:239-246.

5. Ou SH, Kwak EL, Siwak-Tapp C, Dy J, Bergethon K, Clark JW, et al. Activity of crizotinib (PF02341066), a dual mesenchymal-epithelial transition (MET) and anaplastic lymphoma kinase (ALK) inhibitor, in a non-small cell lung cancer patient with de novo MET amplification. J Thorac Oncol. 2011;6:942-946.

6. Lin JJ, Riely GJ, Shaw AT, Targeting ALK. Precision medicine takes on drug resistance. Cancer Discov. 2017;7:137-155.

7. Reck M, Rodrígues-Abreu D, Robinson AG, Hui R, Csőszi T, Fülöp A, et al. Pembrolizumab versus chemotherapy for 
PD-L1-positive non-small-cell lung cancer. N Engl J Med 2016; 375:1823-1833.

8. Dong X, Qiu X, Liu Q, Jia J. Endobronchial ultrasoundguided transbronchial needle aspiration in the mediastinal staging of non-small cell lung cancer: a meta-analysis. Ann Thorac Surg. 2013;96:1502-1507.

9. Jain D, Allen TC, Aisner DL, Beasley MB, Cagle PT, Capelozzi VL, et al. Rapid On-Site Evaluation of Endobronchial Ultrasound-Guided Transbronchial Needle Aspirations for the Diagnosis of Lung Cancer: A Perspective From Members of the Pulmonary Pathology Society. Arch Pathol Lab Med. 2018;142:253-262.

10. Wahidi MM, Herth F, Yasufuku K, Shepherd RW, Yarmus L, Chawla M, et al. Technical Aspects of Endobronchial Ultrasound-Guided Transbronchial Needle Aspiration: CHEST Guideline and Expert Panel Report. Chest. 2016;149:816-835.

11. Ofiara LM, Navasakulpong A, Beaudoin S, Gonzalez AV. Optimizing tissue sampling for the diagnosis, subtyping, and molecular analysis of lung cancer. Front Oncol. 2014;4:253.

12. Cicek T, Ozturk A, Yilmaz A, Aktas Z, Demirag F, Akyurek N. Adequacy of EBUS-TBNA specimen for mutation analysis of lung cancer. Clin Respir J. 2019;13:92-97.

13. Sakakibara R, Inamura K, Tambo Y, Ninomiya H, Kitazono S, Yanagitani N, et al. EBUS-TBNA as a Promising Method for the Evaluation of Tumor PD-L1 Expression in Lung Cancer. Clin Lung Cancer. 2017;18:527-534.e1.

14. Oki M, Saka H, Kitagawa C, Kogure Y, Murata N, Adachi T, et al. Rapid on-site cytologic evaluation during endobronchial ultrasound-guided transbronchial needle aspiration for diagnosing lung cancer: a randomized study. Respiration.. 2013;85:486-492.

15. Chandra S, Chandra H, Sindhwani G. Role of rapid onsite evaluation with cyto-histopathological correlation in diagnosis of lung lesion. J Cytol. 2014;31:189-193.

16. Travis WD, Brambilla E, Noguchi M, Nicholson AG, Geisinger K, Yatabe $\mathrm{Y}$, et al. Diagnosis of lung cancer in small biopsies and cytology: implications of the 2011 International Association for the Study of Lung Cancer/ American Thoracic Society/European Respiratory Society classification. Arch Pathol Lab Med. 2013;137:668-684.

17. Şimon M, Pop B, Toma IL, Vallasek AK, Şimon I. The use of EBUS-TBNA and ROSE in the diagnosis of lung cancer. Rom J Morphol Embryol. 2017;58:79-87.

18. Murakami Y, Oki M, Saka H, Kitagawa C, Kogure Y, Ryuge $\mathrm{M}$, et al. Endobronchial ultrasound-guided transbronchial needle aspiration in the diagnosis of small cell lung cancer. Respiratory Investigation. 2013;52:173-178.

19. Griffin AC, Schwartz LE, Baloch ZW. Utility of onsite evaluation of endobronchial ultrasound-guided transbronchial needle aspiration specimens. Cytojournal. 2011;8:20.

20. Mehta RM, Aurangabadbadwalla R, Singla A, Loknath C, Munavvar M. Endobronchial ultrasound-guided mediastinal lymph node forceps biopsy in patients with negative rapidon-site-evaluation: A new step in the diagnostic algorithm. Clin Respir J. 2020;14:314-319.

21. Simon M, Pop B, Toma L, Vallasek A, Simon I. Endobronchial ultrasound - one year of experience in clinical practice. Clujul Med. 2017;90:188-195.

22. Tomashefski JF Jr, Connors AF Jr, Rosenthal ES, Hsiue IL. Peripheral vs central squamous cell carcinoma of the lung. A comparison of clinical features, histopathology, and survival. Arch Pathol Lab Med. 1990;114:468-474.

23. Mehta HJ, Tanner NT, Silvestri G, Simkovich SM, Shamblin C, Shaftman SR, et al. Outcome of patients with negative and unsatisfactory cytologic specimens obtained by endobronchial ultrasound-guided transbronchial fine-needle aspiration of mediastinal lymph nodes. Cancer Cytopathol. 2015;123:92-97.

24. Nakajima T, Yasufuku K, Suzuki M, Hiroshima K, Kubo R, Mohammed S, etal. Assessment of epidermal growth factor receptor mutation by endobronchial ultrasound-guided transbronchial needle aspiration. Chest. 2007;132:597-602.

25. Masago K, Fujita S, Mio T, Ichikawa M, Sakuma K, Kim $\mathrm{YH}$, etal. Accuracy of epidermal growth factor receptor gene mutation analysis by direct sequencing method based on small biopsy specimens from patients with non-small cell lung cancer: analysis of results in 19 patients. Int J Clin Oncol. 2008;13:442-446.

26. Garcia-Olivé I, Monsó E, Andreo F, Sanz-Santos J, Taron M, Molina-Vila MA, et al. Endobronchial ultrasound-guided transbronchial needle aspiration for identifying EGFR mutations. Eur Respir J. 2010;35: 391-395.

27. Leighl NB, Rekhtman N, Biermann WA, Huang J, MinoKenudson M, Ramalingam SS, et al. Molecular testing for selection of patients with lung cancer for epidermal growth factor receptor and anaplastic lymphoma kinase tyrosine kinase inhibitors: American Society of Clinical Oncology endorsement of the College of American Pathologists/ International Association for the study of lung cancer/ association for molecular pathology guideline. J Clin Oncol. 2014;32:36733679.

28. Zito Marino F, Rossi G, Brunelli M, Malzone MG, Liguori $\mathrm{G}$, Bogina $\mathrm{G}$, et al. Diagnosis of anaplastic lymphoma kinase rearrangement in cytological samples through a fluorescence in situ hybridization-based assay: cytologic smears versus cell blocks. Cancer Cytopathol. 2017;125:303-312.

29. McLeer-Florin A, Moro-Sibilot D, Melis A, Salameire D, Lefebvre C, Ceccaldi F, etal. Dual IHC and FISH testing for ALK gene rearrangement in lung adenocarcinomas in a routine practice: a French study. J Thorac Oncol. 2012;7:348-354.

30. Fernandez-Bussy S, Pires Y, Labarca G, Vial MR. PD-L1 expression in a non-small cell lung cancer specimen obtained by EBUS-TBNA. Arch Bronconeumol. 2018;54:290-292.

31. Biswas A, Leon ME, Drew P, Fernandez-Bussy S, Furtado LV, Jantz MA, et al. Clinical performance of endobronchial ultrasound-guided transbronchial needle aspiration for assessing programmed death ligand-1 expression in nonsmall cell lung cancer. Diagn Cytopathol. 2018;46:378-383.

32. Smith A, Wang H, Zerbo A, Beaudoin S, Ofiara L, Fiset PO, et al. Programmed Death Ligand 1 Testing of Endobronchial Ultrasound-guided Transbronchial Needle Aspiration Samples Acquired For the Diagnosis and Staging of NonSmall Cell Lung Cancer. J Bronchology Interv Pulmonol. 2020;27:50-57. 
33. Evans M, O'Sullivan B, Smith M, Taniere P. Predictive markers for anti-PD-1/PD-L1 therapy in non-small cell lung cancer-where are we? Transl Lung Cancer Res. 2018;7:682690.

34. van der Heijden EH, Casal RF, Trisolini R, Steinfort DP, Hwangbo B, Nakajima T, et al. Guideline for the acquisition and preparation of conventional and endobronchial ultrasound-guided transbronchial needle aspiration specimens for the diagnosis and molecular testing of patients with known or suspected lung cancer. Respiration. 2014;88:500-517.

35. Trisolini R, Cancellieri A, Tinelli C, de Biase D, Valentini I, Casadei G, et al. Randomized Trial of Endobronchial Ultrasound-Guided Transbronchial Needle Aspiration With and Without Rapid On-site Evaluation for Lung Cancer Genotyping. Chest 2015;148:1430-1437.

36. Doxtader E, Yachimiak T, Mukhopadhyay S, Almeida F, Cicenia J, Brainard J, et al. Rapid On-site Evaluation (ROSE) of Endobronchial Ultrasound-guided Fine-needle
Aspiration (EBUS-TBNA) Optimizes Tissue for evaluation of PD-L1 Expression on formalin-fixed Non-small Cell Lung Carcinoma. J Am Soc Cytopathol. 2017;6:S45. doi: 10.1016/j.jasc.2017.06.112

37. Berindan-Neagoe I, Monroig Pdel C, Pasculli B, Calin GA. MicroRNAome genome: a treasure for cancer diagnosis and therapy. CA Cancer J Clin. 2014;64:311-336.

38. Sonea L, Buse M, Gulei D, Onaciu A, Simon I, Braicu C, et al. Decoding the Emerging Patterns Exhibited in Non-coding RNAs Characteristic of Lung Cancer with Regard to their Clinical Significance. Curr Genomics. 2018;19:258-278.

39. Nakajima T, Anayama T, Koike T, Waddell T, Keshavjee S, Kimura H, et al. Simultaneous isolation of total RNA, DNA, and protein using samples obtained by EBUS-TBNA. J Bronchology Interv Pulmonol. 2011;18:301-305.

40. Nakajima T, Yasufuku K, Yoshino I. Current status and perspective of EBUS-TBNA. Gen Thorac Cardiovasc Surg. 2013;61:390-396. 